\section{Descentralização das ações de Vigilância Sanitária nos municípios em Gestão Plena, Estado do Rio de Janeiro}

\section{Decentralization of Health Surveillance actions in cities with local health managment in the State of Rio de Janeiro}

\section{Mirian Miranda Cohen}

Centro de Vigilância Sanitária

Secretaria de Estado da Saúde - RJ

Rua México, 128, $3^{\circ}$ andar, Centro

mcohen@saude.rj.gov.br

\section{Maria de Lourdes de Oliveira Moura}

Centro de Vigilância Sanitária

Secretaria de Estado da Saúde - RJ

Jeane Gláucia Tomazelli

Centro de Vigilância Sanitária

Secretaria de Estado da Saúde - RJ

O Centro de Vigilância Sanitária da Secretaria de Estado da Saúde do Rio de Janeiro pretende, com a descentralização das ações de vigilância sanitária para os Municípios, atender condições mínimas para fortalecer o sistema estadual de Vigilância Sanitária, criando estrutura de apoio ao processo de descentralização. Este trabalho objetiva apresentar o diagnóstico situacional dos órgãos de vigilância sanitária dos municípios em Gestão Plena do Sistema Municipal, discutindo os principais resultados. A metodologia compreende a análise dos 22 órgãos de vigilância sanitária em Gestão Plena do Sistema Municipal, conforme a Norma Operacional Básica 96, no período de julho a dezembro de 2002, através de questionário padrão aplicado pelo Centro de Vigilância Sanitária e de observação participante. Com base nos resultados, conclui-se que a maioria dos órgãos de vigilância sanitária municipais em Gestão Plena do Sistema Municipal, possuem profundas dificuldades técnico-operacionais no desenvolvimento das ações descentralizadas, denotando a fragilidade do processo de descentralização das ações de Vigilância Sanitária no Estado e a necessidade de sensibilizar os gestores para a efetiva estruturação das vigilâncias sanitárias locais, em parceria com o órgão estadual de Vigilância Sanitária.

Palavras-chave: Descentralização. Sistema estadual de vigilância sanitária. Vigilância sanitária local. 
The Health Surveillance Center of the State of Rio de Janeiro intends, with the decentralization of low complexity health surveillance actions for Cities, to meet the minimum requirements to strengthen the state health surveillance system, creating a support structure for the descentraliztion process. The objective of this paper is to present the situational diagnosis of the health surveillance agencies that have adopted the System Full Management. The methodology encompasses the analysis of 22 municipal health surveillance agencies that use the System Full Management, according to the Unified Health System's Basic Operational Norm dated $01 / 96$, in the period from July to December 2002, by means of a standard questionnaire applied by the Health Surveillance Center of the State of Rio de Janeiro, including comments of participants, in which the multiprofessional team interacted with the local services technical team during a technical visit. In addition to collecting data, the team tried to provide guidance as to information techniques and administrative organization. Based on the results, we conclued that most municipal health surveillance agencies with System Full Management have serious technical-operational difficulties in the development of decentralized actions, showing the need to make municipal managers aware of the importance of these actions and to guide them in effectively structuring local health surveillance, in a partnership with the Health surveillance Center of the State of Rio de Janeiro.

Key Words: Decentralization. Health surveillance state system. Local health surveillance.

\section{Introdução}

A descentralização da saúde, uma das diretrizes básicas para a implantação do Sistema Único de Saúde (SUS) no país, tem constituído uma estratégia definidora de nova agenda para o setor.

Ao longo dos anos 90, este processo consolidou a reestruturação da arena decisória em torno da saúde, com transferência de responsabilidades e recursos financeiros para os Estados e municípios.

São diversos os conceitos de descentralização encontrados na literatura. Destacase neste estudo os que consideram descentralização como distribuição de responsabilidades, de recursos e de poder; instrumento que favorece uma maior aproximação com a população; e facilitadora do controle social e fortalecedora da gestão pública na esfera municipall ${ }^{1-4}$.

Neste trabalho, o termo descentralização enfatiza o processo de transferência de recursos e responsabilidades na execução de ações de Vigilância Sanitária (VISA) no âmbito municipal. Parte-se da hipótese que a descentralização, por si só, não garante a mudança no padrão de atenção à saúde.

Acredita-se que, para a descentralização das ações de VISA resultar em maior impacto para a proteção da saúde da população, as responsabilidades entre os gestores do SUS devem ser pactuadas e implementadas de forma integrada entre os entes federativos. Se tal processo é ainda hoje marcado, por um lado, por incertezas quanto a seu alcance e implicações, ele tem, por outro lado, se revelado um campo fértil de experiências municipais inovadoras, seja em relação ao incremento da oferta de serviços, à reorganização do modelo assistencial, ou ainda à revisão das formas de relação público-privado, entre outros aspectos ${ }^{5}$.

Em relação à Vigilância Sanitária, as incertezas e dificuldades se agravam pela própria complexidade de seu objeto, pois suas ações se inserem em todas as práticas médico-sanitárias: promoção, proteção, recuperação e reabilitação da saúde, onde significativa demanda reside na área da Assistência. 
Durante o desenvolvimento do estudo, constatou-se a existência de inúmeros problemas de estruturação das VISAs municipais e o processo recente de organização do órgão estadual.

Este trabalho tem por objetivo discutir o processo de descentralização das ações de vigilância sanitária, a partir da análise do diagnóstico situacional dos órgãos de Vigilância Sanitária dos municípios em Gestão Plena do Sistema Municipal (GPSM) no Estado do Rio de Janeiro, elaborado pelo Centro de Vigilância Sanitária da Secretaria de Estado da Saúde.

Utilizou-se, como recursos metodológicos a pesquisa documental e a análise de dados obtidos em documentos e questionários resultantes do processo de avaliação das ações descentralizadas, que incluiu concepções teóricas de abordagem a partir de levantamento bibliográfico específico.

O diagnóstico foi efetuado através da aplicação de questionário* padrão em visita de supervisão às VISAs municipais, por equipe multiprofissional, formada por representantes de diversos setores do CVS/SES-RJ. No processo de avaliação dos órgãos locais de Vigilância Sanitária foram destacados os seguintes aspectos: adequação da estrutura física; aquisição de equipamentos; existência de equipe multiprofissional; vínculo dos profissionais; capacitação profissional; existência de cadastro dos estabelecimentos sujeitos às ações de Vigilância Sanitária; apresentação de ato formal de criação do órgão de Vigilância Sanitária; elaboração de relatório de inspeção; realização de coleta de amostras de produtos; existência de instrumentos necessários ao desempenho das atividades (conjunto de termos, roteiro de inspeção e Código Sanitário Municipal) e condições de arquivamento de processos.

Na elaboração do diagnóstico situacional dos órgãos de Vigilância Sanitária pelo CVS/ SES-RJ, estes aspectos foram considerados suficientes para se conhecer as condições de desempenho das ações locais de Vigilância
Sanitária. Cabe a análise da suficiência ou não destes quesitos como base metodológica de avaliação do processo de descentralização.

Como metodologia analítica neste estudo, buscou-se confrontar os resultados encontrados no mencionado diagnóstico com o referencial teórico afeto à área e a adesão ao processo de descentralização, onde será enfatizada a relação entre estar em GPSM e ter condição de desenvolver ações básicas de Vigilância Sanitária.

A relevância deste trabalho reside na potencial contribuição à orientação metodológica do processo de avaliação das ações descentralizadas, onde se busca a reorientação do modelo tradicional de Atenção à Saúde, favorecendo a formulação intersetorial de um conjunto de novas práticas sanitárias.

Assim, na perspectiva de refletir sobre a descentralização e Vigilância Sanitária no SUS, este artigo tem como propósito, à luz das questões das Políticas Públicas de Saúde, discutir a descentralização das ações de Vigilância Sanitária no Estado do Rio de Janeiro, enfocando os municípios em Gestão Plena do Sistema Municipal (GPSM).

\section{A VISA no SUS: descentralização e perspectivas no Estado do Rio de Janeiro}

No texto constitucional, a VISA é explicitada como ação específica do setor saúde. Entretanto, diferentemente de outras áreas fiscais, a Saúde não tem o seu poder de polícia administrativa tratado como matéria constitucional. A Vigilância Sanitária, porém, campo da Saúde Pública na qual este poder é exercido, está regulamentada na Lei Federal no $8080 / 90^{6}$, onde em seu artigo $6^{\circ}$ a vigilância sanitária é definida como "um conjunto de ações capaz de eliminar, diminuir ou prevenir riscos à saúde e de intervir nos problemas sanitários decorrentes do meio ambiente, da produção e circulação de bens e da prestação de serviços de interesse da saúde".

*O questionário foi desenvolvido pela equipe do NPDI/CVS/SES-RJ, tendo como referência o instrumento "Levantamento da Situação dos Órgãos de Vigilância Sanitária dos municípios" ${ }^{15,16}$, e as Resoluções SES 562/906 e 1262/987, assim como a NOAS/ SUS $01 / 2002^{8}$. 
Várias iniciativas vêm sendo desenvolvidas na busca de transformar o arcabouço jurídico-legal do SUS em realidade, sendo o seu processo de regulamentação conduzido, principalmente, por instrumentos legais: as Normas Operacionais que tratam especialmente dos aspectos da divisão de responsabilidades, das relações entre gestores e dos critérios de transferências de recursos federais para Estados e municípios, ou seja, da descentralização.

"Dentre as diretrizes e princípios que orientam o SUS, pode-se afirmar que a descentralização é aquele cujos processos e resultados têm sido mais estudados e acompanhados, e vários são os desafios e avanços registrados"7.

Como instrumento de Regulação do processo de descentralização, ao longo dos 15 anos do Sistema Único de Saúde, foram editadas quatro Normas Operacionais: 1991/ 1992; 1993; 1996 e 2001/2002. Estas abordam especificamente alguns aspectos do desenvolvimento das políticas de saúde e não de todo o sistema, e apresentam diferenças fundamentais que refletem o momento político em que foram elaboradas e a intensidade do processo de pactuação entre gestores para sua formulação.

Assim, a Proteção e a Promoção à Saúde, previstas constitucionalmente, não são mencionadas na NOB SUS 01/91 e nem na sua substituta, a NOB SUS 01/92, que omitem a Vigilância Sanitária e a Epidemiológica, não contemplando o princípio da integralidade do sistema. Contudo, a descentralização está em foco, e em 1990, no Estado do Rio de Janeiro, inicia-se formalmente o processo de municipalização na área de VISA, com a publicação da Resolução SES 562/90', que transfere aos municípios a responsabilidade de execução de ações de baixa complexidade em Vigilância Sanitária.

Esta dispõe sobre o controle sanitário do comércio de alimentos, drogas, insumos farmacêuticos e correlatos, cosméticos, saneantes domissanitários (apreensão de produtos por determinação federal e estadual) e o controle sanitário das construções em geral pelas Secretarias Municipais de Saúde.
Entretanto, como os mecanismos de regulação e financiamento não foram desenvolvidos e as condições para sua implementação não estavam estabelecidas, o processo de descentralização das ações básicas de Vigilância Sanitária foi incipiente e recebido apenas burocraticamente pela maioria dos municípios.

A NOB SUS 01/93, assim como sua antecessora, não teve impacto direto sobre a área de Vigilância Sanitária, apesar de sua importância inquestionável na implementação do processo de descentralização das ações de saúde. Como principais implementações na NOB SUS 01/93, pode-se apontar o início do repasse "fundo a fundo" para municípios habilitados e o estabelecimento das instâncias intergestoras (Comissão Intergestores Bipartite - CIB e Comissão Intergestores Tripartite - CIT).

Mas, ao descrever as responsabilidades que os Estados e municípios devem assumir ao pleitearem as diferentes condições de gestão, contemplou prioritariamente as atividades ambulatoriais e hospitalares, apesar de fazer referência à Vigilância Sanitária ${ }^{9}$.

Sem indicação dos mecanismos de operacionalização das ações de VISA, com indefinição das responsabilidades dos governos federal, estadual e municipal na sua execução e sem indicação de novas possibilidades de financiamento para a área, a VISA não obteve respaldo na NOB SUS 01/93 para avançar no processo de descentralização. Dando continuidade à normalização do processo de descentralização, é editada a NOB SUS $01 / 96^{10}$, que tem como característica principal a implementação de uma série de regras, com a finalidade de reforçar e consolidar a capacidade de gestão do sistema por parte do poder público e redefinir as responsabilidades dos Estados, Municípios e União em relação às suas atribuições no Sistema Único de Saúde. Confere maiores responsabilidades ao âmbito municipal, ao mesmo tempo em que aponta para uma reordenação do modelo de atenção à saúde. Além disso, catalisa o processo de descentralização, definindo novas formas de gestão (Gestão Plena da Atenção Básica e Ges- 
tão Plena do Sistema). A diferença entre as duas condições de gestão diz respeito ao grau de responsabilidades assumidas pelo gestor.

Contudo, ao não diferenciar o conjunto de atividades e procedimentos de Vigilância Sanitária correspondentes às ações de baixa, média e de alta complexidade, a NOB SUS 01/96 não viabiliza a operacionalização imediata das responsabilidades específicas detalhadas para cada tipo de gestão. A portaria SAS/MS no 18 de 21/01/99 ${ }^{11}$ traz esse detalhamento, contudo a mesma vem sendo sistematicamente questionada.

A NOB/96 inova ao introduzir o Piso Básico de Vigilância Sanitária (PBVS), inserido como parte variável do Piso de Atenção Básica, assim como ao descrever, mesmo de forma genérica, as atribuições de cada gestor nas três esferas de governo no tocante à Vigilância Sanitária.

Sem questionar o poder indutor da norma, pode-se afirmar que o cenário político em que ela se constituiu, quando escândalos freqüentes relacionados principalmente à comercialização irregular de medicamentos fraudados eram levados a público, contribuiu para que o período de sua implementação coincidisse com a deflagração de novas estratégias de reorientação do processo de descentralização das ações de Vigilância Sanitária no Estado do Rio de Janeiro.

No período de implementação da NOB 01/96, ou seja, a partir de 1998, a Secretaria de Estado da Saúde do Rio de Janeiro, através de seu serviço de Vigilância Sanitária, conclamou os municípios a avançarem no processo de descentralização. Naquele momento, somente 26 municípios aderiram oficialmente à proposta, mas apenas 14 têm tido condições de cumprir o que foi pactuado.

Todos os municípios que aderiram à proposta estão listados na Resolução SES 1262/ $98^{12}$, que lhes delega competência para executar ações de Vigilância Sanitária nos estabelecimentos de interesse da saúde pública (vigilância sanitária de serviços de saúde sem internação e vigilância sanitária de estabelecimentos de comércio farmacêutico); conceder licença de funcionamento e/ou revalidá-la; cassar licença de funcionamen- to; e fiscalizar outros estabelecimentos sujeitos a ação de VISA.

Cabe ressaltar que no processo de habilitação a uma das formas de gestão no SUS, na análise dos pleitos municipais, a Vigilância Sanitária não foi contemplada como um dos quesitos de avaliação. Este fato provocou a inexistência de correlação entre tipo de gestão do SUS e estruturação dos órgãos de Vigilância Sanitária.

Na prática encontra-se VISAs municipais em GPSM sem condições mínimas para o desempenho de suas ações, e, em menor número, VISAs em GPAB plenamente estruturadas para desenvolverem adequadamente suas ações. Mas, certamente, espera-se que as Secretarias Municipais em GPSM, avaliadas como possuidoras de efetivo Sistema Local de Saúde, tenham melhores condições para estruturar seus órgãos de Vigilância Sanitária.

Em tese, cabe ao Estado a execução de ações de VISA de baixa, média e/ou alta complexidade, apenas quando o município não está habilitado a executá-las, de acordo com as condições de gestão previstas na NOB SUS 01/96. Na prática, executar ações de vigilância sanitária de alta complexidade requer capacidade técnica e recursos humanos especializados, exigência que muitos municípios de médio e pequeno porte, não têm condições de manter, pelo menos por enquanto, ficando o Estado como o principal executor dessas ações.

Em síntese, a exemplo da norma anterior, a NOB SUS 01/96 engloba de modo frágil a área de VISA entre as responsabilidades das diferentes condições de gestão, embora inove ao propor mecanismos de financiamento para o custeio dessas ações.

Para atender os objetivos de fortalecer as instâncias estaduais e dar continuidade ao processo de descentralização, após discussão e levantamento das dificuldades apontadas pelas três esferas de governo, foi publicada mais uma Norma Operacional pelo Ministério da Saúde: a Norma Operacional de Assistência à Saúde (NOAS SUS 01/02) ${ }^{13}$.

ANOASSUS 01/02 avança ao propor, para a sistematização do processo de habilitação 
dos municípios, alguns requisitos relativos à Vigilância Sanitária. O desafio imposto à NOAS-SUS 01/02 é se configurar em mecanismo indutor do processo de descentralização, favorecendo a integralidade do sistema e respeitando as diversidades regionais.

Há duas condições de gestão previstas, nas quais os municípios podem se habilitar: Gestão Plena da Atenção Básica Ampliada (GPABA) e Gestão Plena do Sistema Municipal (GPSM). Para cada condição de gestão há um conjunto de prerrogativas, responsabilidades, requisitos e instrumentos de comprovação que deverão ser cumpridos pelos municípios que desejarem se adequar à Norma.

Com relação à Vigilância Sanitária, para que o município possa se habilitar à condição de GPABA e GPSM, dentre outros requisitos deverá, respectivamente, comprovar condições para o desenvolvimento das ações básicas de VISA e para a execução de ações de maior complexidade nas áreas de Vigilância Sanitária.

No processo de avaliação da capacidade dos municípios para o desenvolvimento das ações de Vigilância Sanitária, a Secretaria de Estado da Saúde (SES-RJ), considerando o estágio atual do processo de descentralização, estabeleceu, através do CVS/SESRJ, o seguinte critério de avaliação das VISAs municipais para habilitação à NOAS 01/2002:

- municípios que pleitearem a habilitação em GPSM: desenvolver o conjunto de ações Básicas de VISA, conforme a Portaria SAS 18/99, além de estarem aptos a pleitearem a pactuação de ações de média complexidade, de acordo com a Portaria MS $2473 / 2003^{14}$, e além também de apresentarem em seus pleitos de habilitação Declaração Conjunta assinada pelos gestores estadual (SES) e municipal (SMS), explicitando as responsabilidades específicas do município e do Estado nas ações de vigilância sanitária; $\mathrm{e}$

- municípios que pleitearem a habilitação em GPABA: buscar atender as ações básicas, de acordo com a Portaria SAS 18/ 99, além de apresentarem em seus pleitos de habilitação Declaração Conjunta da SES e da SMS, explicitando as res- ponsabilidades específicas do município e do Estado nas ações de vigilância sanitária.

Com a finalidade de constatar a capacidade municipal para desenvolver ações de VISA e colaborar com o nível local no processo de organização para habilitação à NOAS, o CVS/SES-RJ encaminha aos municípios uma equipe multiprofissional que tem a responsabilidade de avaliar a VISA municipal, aplicando questionário padrão específico.

O resultado desta avaliação não permitiu constatar correlação entre tipo de gestão no SUS e estruturação dos órgãos de VISA. Entretanto, é evidente o propósito do CVS/ SES-RJ de utilizar este processo de habilitação à NOAS para reorientar a estruturação dos órgãos de Vigilância Sanitária para o desenvolvimento das ações descentralizadas, sendo a pactuação na elaboração da Declaração Conjunta importante estratégia priorizada.

Como se pôde perceber, a NOAS traz novos critérios para habilitação de Estados e municípios e engendra novos desafios à gestão estadual no processo de condução da descentralização.

Várias têm sido as iniciativas, no âmbito da Vigilância Sanitária, para apoiar o processo de descentralização no Estado do Rio de Janeiro. Por outro lado, é fato que ao ter importante função executora das ações de Vigilância Sanitária, o CVS/SES-RJ apresenta maior dificuldade para assumir seu papel coordenador e regulador do Sistema, enfrentando obstáculos para cumprir a agenda de apoio aos órgãos municipais no desenvolvimento das ações descentralizadas, mesmo sem atingir cobertura de $100 \%$ nas inspeções dos estabelecimentos sujeitos às ações de Vigilância Sanitária.

A forma encontrada para superar estes obstáculos foi priorizar a implementação do processo de descentralização, tendo como parâmetro a distribuição de responsabilidades de acordo com o risco sanitário atribuído ao procedimento.

Para Lucchese ${ }^{12}$, deve existir a avaliação do risco e a gerência do risco que são fundamentais para a estruturação das interven- 
ções do Estado no campo da regulação do risco sanitário. Porque a análise de risco avalia a probabilidade de acontecer um efeito adverso por um agente (químico, físico, biológico e outros), processos industriais, tecnologia ou processo natural; e, no campo sanitário, os efeitos adversos são quase sempre relacionados a algum dano à saúde, a doenças e, até mesmo, à morte.

Entretanto, um dos maiores desafios para a ação regulatória do Estado moderno na área sanitária é a avaliação do risco das novas tecnologias, sejam elas substâncias, aparelhos ou serviços.

Para que se possa atuar nesta lógica éimprescindível que o município assuma, pelo menos, o conjunto de procedimentos que são básicos, menos complexos, mas fundamentais para proteção da Saúde da população.

Desta forma, a Vigilância Sanitária estadual poderá cumprir seu efetivo papel coordenador e regulador, mesmo executando os procedimentos de maior complexidade em Vigilância Sanitária. Não basta fiscalizar burocraticamente; é necessário usar a técnica apropriada e identificar quais são os pontos críticos naquela unidade, seja num estabelecimento de saúde ou em qualquer outro sujeito à Vigilância Sanitária, buscando Boas Práticas na fabricação, comercialização ou prestação de serviços. Daí a necessidade de divisão solidária de responsabilidades entre os entes federativos e de capacitação permanente dos profissionais.

A perspectiva no Estado do Rio de Janeiro é de avanço no processo de descentralização e que o Estado possa apoiar as VISAs locais no desenvolvimento de suas atividades, pactuando a Harmonização dos procedimentos, capacitando e supervisionando as equipes, com trabalho de verificação de falhas de procedimentos, análise de processos e revisão dos processos de trabalho. Em síntese, este estudo permitiu o levantamento das seguintes perspectivas para o processo de descentralização do Estado:

- Descentralização de Ações de média complexidade-As estratégias priorizadas pelo CVS/SES-RJ, neste momento, para a implementação do processo de descen- tralização das ações de média complexidade, são: discussão constante de temas afetos á VISA, sobretudo aqueles considerados estratégicos para o processo de descentralização, no COSEMS e na Câmara técnica da CIB; reuniões mensais com os 28 municípios aptos a assumir as ações de média complexidade; formação de grupos temáticos de trabalho; realização de oficinas regionais de apoio ao processo de descentralização; capacitação dos profissionais de VISA; e estruturação dos órgãos de VISA municipal, com repasse de equipamentos e insumos.

Foram formados sete grupos de trabalho temáticos, com representantes das VISAs Municipais e coordenados por técnicos da VISA Estadual. Esses grupos têm como objetivo a padronização dos procedimentos, sejam de baixa ou média complexidade, e abordam as seguintes áreas priorizadas no processo de descentralização:

- VISA em Unidades Básicas de Saúde (inclui os estabelecimentos odontológicos);

- VISA em Estabelecimentos de Atenção ao Idoso;

- VISA em Laboratórios Clínicos e postos de coleta;

- VISA em Estabelecimentos de Radiodiagnóstico;

- VISA em Farmácia de manipulação;

- VISA em Cozinhas Industriais e em Indústrias de Alimentos dispensados de registro;

- VISA em salão de beleza, esteticistas, academias de ginásticas e congêneres.

O objetivo que impulsiona a constituição destes grupos de trabalho é a perspectiva de harmonização dos ritos e procedimentos em cada área de trabalho. Espera-se desta forma possibilitar melhor compreensão das ações desenvolvidas pelos técnicos, setor regulado e a população, e favorecer o processo de avaliação de desempenho e ações de suporte por parte do órgão estadual.

- Descentralização do conjunto de ações de Vigilância Sanitária de baixa complexidade para $100 \%$ dos municípios-A ou- 
tra perspectiva do trabalho do CVS/SES/ RJ é que, em 2004, os 92 municípios existentes no Estado do Rio de Janeiro estruturem minimamente seus serviços de Vigilância Sanitária e passem a assumir a execução das ações básicas de Vigilância Sanitária. Propõe as seguintes estratégias: articulação constante com as Vigilâncias Sanitárias municipais e o COSEMS; implantação da Câmara Técnica de Vigilância Sanitária da CIB-RJ; fortalecimento das estruturas internas do CVS/SES-RJ, com criação e implementação de setores estratégicos; formulação de Programas Estaduais; capacitação de profissionais de Vigilância Sanitária e gestores; apoio à estruturação das Vigilâncias Sanitárias locais; comunicação sistêmica (através da Internet, fax, telefone, reuniões; encontros informais etc.); e realização de oficinas regionais de apoio ao processo de descentralização.

Neste trabalho, a ótica percebida no CVS/ SES-RJ foi a de intenso processo de pactuação na organização do sistema estadual de Vigilância Sanitária, com a incorporação do papel executor dos municípios, possibilitando ao órgão estadual assumir o seu papel regulador e coordenador do sistema, entendendo que a sua função é proteger a saúde da população e que essa responsabilidade deve ser solidariamente compartilhada pela União, os Estados e os municípios.

O desafio é incorporar os avanços obtidos no SUS no que se refere à descentralização aos serviços de Vigilância Sanitária, sem configurar um processo meramente burocrático e percebendo a descentralização em Vigilância Sanitária como ação inerente a todas as atividades, que não deve ser entendida como Programa pontual, mas como estratégia operacional a ser freqüentemente aprimorada.

\section{As vigilâncias sanitárias em GPSM no Estado do Rio de Janeiro}

O diagnóstico dos órgãos de Vigilância Sanitária municipal no Estado do Rio de Janeiro, dos 22 municípios em GPSM, permite uma análise critica do processo de descentralização das ações de VISA no Estado.

Este tópico apresenta análise dos principais resultados do processo de avaliação das VISAs municipais em GPSM no desempenho de suas ações, realizada pelo CVS-SES/RJ.

As informações foram organizadas em 5 grupos: Estrutura do Serviço - estrutura física, equipamentos, cadastro dos estabelecimentos e instrumentos para o desenvolvimento das ações de VISA; Recursos Financeiros - recursos do Piso de Atenção BásicaPAB-VISA e recursos arrecadados pelas VISAs; Recursos Humanos - perfil dos responsáveis pela VISA, equipe multiprofissional e escolaridade dos técnicos; Capacitação em VISA/ Ações Educativas - capacitação profissional e ações de Informação, Educação e Comunicação em VISA e Ações de VISA - Inspeções realizadas pelas VISAs, utilização de instrumentos nas ações, rotinas implantadas e dificuldades levantadas pelas VISAs para o desenvolvimento das ações.

Estrutura do Serviço - A precária infraestrutura organizacional e operacional prevalece nas VISAs municipais, principalmente quanto ao espaço físico (36\% das VISAs possuem local adequado para o desenvolvimento das atividades, somente $64 \%$ têm local próprio para arquivamento dos processos, e apenas 59\% têm a posse dos mesmos). Outro aspecto importante relaciona-se à posse de equipamento de informática, quando $95 \%$ das VISAs possuem microcomputadores, mas $46 \%$ não tem acesso à Internet.

Recursos Financeiros - Com relação à arrecadação própria, apenas onze municípios (50\%) arrecadam, os quais freqüentemente não recebem o repasse da verba pela Prefeitura.

O desconhecimento da maioria dos coordenadores sobre o tipo de gestão municipal e o montante do Piso Básico de Vigilância Sanitária (54\% desconhecem o valor) sugere dificuldades no exercício de suas atribuições e a falta de transparência na aplicação do recurso.

Recursos Humanos - Foi identificado reduzido tempo de permanência dos res- 
ponsáveis pelas VISAs municipais na coordenação, o que, associado à falta de vínculo estável, causa a descontinuidade dos trabalhos (50\% dos Coordenadores estão na atividade há menos de 01 ano e $86 \%$ há menos de 1,5 ano).

Do total de coordenadores das VISAs em GPSM, quatorze (64\%) não integram o quadro efetivo do órgão e quatro (18\%) não possuem nível superior. Com relação à formação dos coordenadores, a maior parte (72 \%) é constituída de médicos veterinários.

Vale lembrar que as ações de VISA têm sido prejudicadas em função da rotatividade dos profissionais lotados no órgão de VISA (68\% não são do quadro efetivo), ocasionando a demissão de profissionais já capacitados por ocasião de mudança na gestão.

O levantamento sobre a existência de equipe multiprofissional*, revelou que 8 municípios em GPSM (36\%) não possuem esta equipe. Cabe ressaltar que ter equipe multiprofissional não significa necessariamente tê-la em quantidade suficiente para o desenvolvimento das ações de VISA, nem que o conjunto de profissionais seja do quadro efetivo e esteja capacitado, pois se assim fosse o total de municípios seria considerado inadequado neste critério.

Desta forma, a formação da equipe multidisciplinar e sua capacitação continua sendo um grande desafio a ser enfrentado na descentralização das ações de VISA, além da necessidade de se estabelecer procedimentos operacionais padrão, visando à harmonização dos processos e das práticas no estado.

Capacitação em VISA/Ações Educativas - Contrapondo-se ao significativo volume de eventos educativos promovidos pela SES às VISAs municipais em 2002, os coordenadores relatam necessidade de capacitação de sua equipe em VISA. Esta oposição expressa a precariedade do vínculo dos profissionais e, conseqüentemente, a sua grande rotatividade, que provoca descompasso entre o elevado número de cursos ofertados e o de profissionais atualmente capacitados, ocasionando perda dos esforços de capacitação empreendidos pela CVS/SES/RJ. Ainda assim, os cursos promovidos pelo Estado surgem como principal estratégia de capacitação.

A maior parte das Vigilâncias Sanitárias (82\%) já recebeu capacitação promovida pelo Estado. A capacitação promovida pelo próprio município ainda é reduzida, tendo sido realizada por apenas seis municípios (27\%).

Ações de VISA - A baixa implantação de rotinas nas atividades essenciais, a baixa existência de roteiros de inspeção (27\% das VISAs não possuem roteiro para nenhuma área) $\mathrm{e}$ a não elaboração de relatórios de inspeção colocam em questionamento a qualificação das ações e demonstram a falta de padronização das atividades. $100 \%$ das VISAs Municipais em GPSM não possuem o conjunto de roteiros necessários ao desenvolvimento de suas ações. Parcela importante dos municípios (18\%) não possui Código Sanitário e os existentes necessitam de atualizações. Muitos municípios (45\%) não possuem qualquer forma de cadastro dos estabelecimentos sujeitos à VISA, enquanto outros o possuem desatualizado, muitas vezes cópia do cadastro da Secretaria Municipal da Fazenda.

$\mathrm{Na}$ análise de utilização de roteiros de inspeção e utilização de indicadores para o acompanhamento das ações de VISA, verificou-se que dezesseis municípios (73\%) utilizam pelo menos um roteiro de inspeção, enquanto apenas cinco municípios (23\%) fazem uso de indicadores.

Este elenco de informações expressa principalmente carências estruturais historicamente determinadas no sistema de Vigilância Sanitária, cujo equacionamento depende de decisões políticas alicerçadas em conhecimento da área, compromisso, transparência, responsabilização e governabilidade, assim como necessita de recursos orçamentários significativos.

Com relação às principais dificuldades identificadas pelas VISAs municipais em

*Equipe multiprofissional: composta de 3 ou mais categorias profissionais de nível superior das profissões previstas na Resolução SES N 1.262 de 08 de dezembro de 1998 (farmacêuticos, médicos, enfermeiros, odontólogos, arquitetos e demais profissionais em quantidade suficiente para a execução de inspeção sanitária). 
GPSM no desenvolvimento de suas ações, a falta de profissionais efetivos foi a mais identificada (77\%), seguida da falta de infraestrutura operacional/equipamentos (73\%), a falta de veículos (32\%) e a ausência de capacitação técnica (32\%).

A falta de equipe mínima é um dos grandes entraves ao avanço do processo de descentralização. Torna-se prioritária a admissão de profissionais por concurso público, para evitar a alta rotatividade e problemas de legalidade no exercício da função da fiscalização sanitária, que exige fé pública. Há que se considerar, sem discutir a sua suficiência, que são previstos nos SUS recursos financeiros para incentivo às ações de Vigilância Sanitária, que ao se deparar com a realidade de estruturação dos órgãos de VISA municipais no Estado do Rio de Janeiro, podem ter sua aplicação questionada.

Cabe ressaltar que, no processo de descentralização das ações de Vigilância Sanitária de baixa complexidade, o repasse de recursos financeiros do âmbito estadual para o municipal não foi previsto. A transferência que ocorre é através do repasse de recursos materiais às VISAs municipais, como equipamentos de informática, veículos etc.

Enfim, pode-se sugerir a existência de recursos que, se aplicados efetivamente no órgão de VISA municipal, poderão refletir em alguma estrutura na execução de ações de VISA. É necessário atentar para a necessidade permanente de incentivo, mas sobretudo de regulação no processo de descentralização das ações de VISA. Este conjunto de dados reforça a fragilidade na estruturação das ações de VISA no nível local, visto que, dos municípios em GPSM, é esperado que tenham melhores condições para o desempenho de suas funções. Refletem também as dificuldades impostas ao processo de descentralização das ações de Vigilância Sanitária.

\section{Considerações Finais}

A discussão sobre a descentralização das ações de VISA tem suscitado diversas polêmicas relativas ao seu modelo e procedência. $\mathrm{O}$ fato de ser atribuição de governo, ou seja, de ser atividade restrita ao setor público, faz com que a implementação do processo de descentralização das ações de VISA seja distinta do proposto para a área da assistência, onde a compra de serviços pelo setor público ao setor privado é uma alternativa para garantir a prestação do serviço.

Em cada Estado, o alcance da descentralização é diretamente proporcional ao poder de ação do gestor, ou seja, quanto mais sintonizados estiverem os poderes executivos, maior será a extensão da municipalização daquele Estado. Logicamente, fatores socioeconômicos e culturais também influenciam este processo.

Tanto as ações dos executivos estaduais como as estratégias implementadas pelo governo federal são determinantes para se municipalizar as atribuições de gestão. Portanto, a precariedade política nesta área acarretará um pesado ônus para a administração municipal, que, sozinha, na maioria dos casos, não tem como arcar com os custos ${ }^{15}$.

Ao analisar o processo de descentralização das ações de Vigilância Sanitária no Estado do Rio de Janeiro, a partir do diagnóstico situacional dos órgãos de VISA em GPSM, percebe-se grande precariedade na estruturação dos órgãos e recente movimento estadual no apoio ao desenvolvimento das ações descentralizadas. Isto reforça a necessidade de mecanismos de indução, mas sobretudo de regulação, no processo de descentralização das ações de VISA.

Ainda segundo Arretche ${ }^{15}$, a descentralização depende da implementação de estratégias deliberadas dos níveis mais abrangentes de governo, que pretendem transferir responsabilidades ao nível local, a fim de favorecer a adesão à proposta.

Porém, cabe aos municípios um conjunto de ações básicas fundamentais para que $o$ processo possa ser implementado. No Estado do Rio de Janeiro, a falta de estruturação dos órgãos locais de VISA exige do gestor municipal medidas indelegáveis para que a descentralização se efetive, como, por exemplo, alocar recursos humanos no setor. Os resultados deste estudo apontam para a relevância da estratégia de sensibilização de 
gestores e para a importância do seu comprometimento com a efetiva estruturação das VISAs municipais em parceria com o Estado.

Ao gestor estadual cabe adotar medidas que favoreçam a adesão ao processo de descentralização, incluindo um conjunto de estratégias de apoio ao município na execução de suas atividades, onde se insere o repasse de recursos.

Os critérios adotados no processo de avaliação das ações descentralizadas demonstram suficiência, mas carecem de estudos que permitam a definição de score e parâmetros de avaliação.

Os dados revelam a ausência de correspondência entre estar em GPSM e estruturação do órgão de Vigilância Sanitária, denotando a não priorização das ações de VISA no contexto da descentralização.

Cabe ainda definir, junto aos gestores das diferentes esferas de governo, estratégias que permitam a padronização das atividades, respeitando-se a legislação e a competência para o seu desenvolvimento.

Ao analisar o processo de descentralização das ações de VISA no Estado do Rio de Janeiro, observa-se que o CVS/SES-RJ é o executor preferencial das atividades de Vigilância Sanitária no Estado, sendo os municípios colaboradores neste processo. Situação que obstaculiza o cumprimento efetivo do papel coordenador, regulador, normalizador, capacitador e financiador do órgão estadual.

O quadro observado reforça a importância da ação direta do CVS/SES-RJ e do maior investimento dos gestores municipais, frente à precária estruturação das VISAs locais, para que se efetive o processo de descentralização das suas ações com a qualidade esperada.

A estratégia para a implementação do sistema estadual de Vigilância Sanitária enfocou em primeiro plano a estruturação do órgão estadual, voltando-se mais recentemente para os laboratórios de referência e as VISAs municipais. Logo, este processo é recente e carece de constante avaliação e reorientação.

Neste percurso, além da dimensão física, o maior investimento foi feito na admissão de novos profissionais de diversas categorias e em sua capacitação; na revisão dos processos de trabalho; e na criação de setores estratégicos.

Atualmente, entre as atividades priorizadas estão as de suporte ao processo de descentralização, onde se incluem o acompanhamento e a avaliação das ações descentralizadas.

Nota-se grande importância dada à aplicação das ferramentas do planejamento e da epidemiologia na reorientação dos paradigmas da Vigilância Sanitária, que evoca a necessidade de intenso diálogo entre os órgãos estadual e municipal de VISA, na compreensão da descentralização como ação estratégica e não como programa pontual.

No Estado do Rio de Janeiro, o grande desafio para a implementação do processo de descentralização tem sido provocar a estruturação das VISAs municipais, com formação de equipe multiprofissional mínima e sua capacitação, além de buscar, de forma articulada com os municípios, harmonizar os ritos e procedimentos estaduais, principalmente para a execução de ações de VISA de Serviços de Saúde pelas instâncias municipais.

\section{Referências}

1. Costa EA, Rozenfeld S. Constituição da Vigilância Sanitária no Brasil. Fundamentos da Vigilância Sanitária. Rio de Janeiro: Ed. FIOCRUZ; 2000.

2. Lucchese G. Globalização e Regulação Sanitária. Os Rumos da VISA no Brasil. Tese de doutorado. Rio de Janeiro: ENSP/FIOCRUZ; 2001.
3. Cohen MM. O Modelo Assistencial no SUS - Niterói: Conflito e Pactuação Política no processo de Integração do Programa Médico de Família. Tese de mestrado em Saúde Pública - Sub-área de Políticas Públicas e Saúde. Rio de Janeiro: ENSP/FIOCRUZ; 2001. 
4. De Seta MH, Silva JAA. A Gestão em Vigilância Sanitária. Curso de atualização em gestão da VISA para dirigentes do RJ-2003. ENSP/FIOCRUZ, 2003.

5. Senna MCM, Cohen MM. Modelo Assistencial e Estratégia Saúde da Família no nível local: Análise de uma experiência. Ciênc Saúde Coletiva - ABRASCO 2002; 7(3); 523-535.

6. Congresso Nacional. Lei Federal no 8080 de 19 de setembro de 1990. Brasília, DF.

7. Cohen MM, Lima JC, Pereira CRA. Normas Operacionais do SUS e a Vigilância Sanitária. Curso de atualização em gestão da VISA para dirigentes do RJ-2003. ENSP/FIOCRUZ, 2003.

8. Secretaria de Estado da Saúde do Rio de Janeiro. Resolução SES 562 de 1990. Rio de Janeiro.

9. Lucchese PTR. A Vigilância Sanitária, segundo as normas operacionais básicas do Ministério da Saúde. In: Rozenfeld S (org.) Fundamentos da Vigilância Sanitária. Rio de Janeiro: Ed. FIOCRUZ; 2000.
10. Norma Operacional Básica, NOB-SUS 01/96. Brasília, DF.

11. Ministério da Saúde. Portaria SAS/MS nº 18 de 1991. Brasília, DF.

12. Secretaria de Estado da Saúde do Rio de Janeiro. Resolução SES 1262 de 1998. Rio de Janeiro.

13. Ministério da Saúde. Portaria $n^{\circ} 373 / G M$. Norma Operacional de Assistência à Saúde 01/2002. Brasília, DF.

14. Ministério da Saúde. Portaria no 2.473 de 29 de dezembro de 2003. Brasília, DF.

15. Arretche MTS. Políticas Sociais no Brasil: descentralização em um Estado federativo. Rbcs 1999; 14(40); 111-41.

16. Reis LC, Lima LCW. Levantamento da Situação dos Órgãos de Vigilância Sanitária dos municípios. Brasília: ANVISA; 2000. Digital. www.anvisa.gov.br

recebido em: $17 / 11 / 03$

versão reformulada apresentada em: 10/05/04 aprovado em: 20/09/04 